\title{
Control of recoil losses in nanomechanical SiN membrane resonators
}

\author{
A. Borrielli, ${ }^{1,2}$ L. Marconi, ${ }^{3,4}$ F. Marin,,${ }^{3,45,6}$ F. Marino, ${ }^{3,4}$ B. Morana,,${ }^{1,7}$ G. Pandraud, ${ }^{7}$ A. Pontin, ${ }^{4,5}$ G. A. Prodi,,${ }^{2,8}$ \\ P. M. Sarro, ${ }^{7}$ E. Serra, ${ }^{2,7}$ and M. Bonaldi ${ }^{1,2, *}$ \\ ${ }^{1}$ Institute of Materials for Electronics and Magnetism, Nanoscience-Trento-FBK Division, 38123 Povo (TN), Italy \\ ${ }^{2}$ Istituto Nazionale di Fisica Nucleare, TIFPA, 38123 Povo (TN), Italy \\ ${ }^{3}$ CNR-INO, L.go Enrico Fermi 6, 50125 Firenze, Italy \\ ${ }^{4}$ INFN, Sezione di Firenze, Via Sansone 1, 50019 Sesto Fiorentino (FI), Italy \\ ${ }^{5}$ Dipartimento di Fisica e Astronomia, Università di Firenze, Via Sansone 1, 50019 Sesto Fiorentino (FI), Italy \\ ${ }^{6}$ LENS, Via Carrara 1, 50019 Sesto Fiorentino (FI), Italy \\ ${ }^{7}$ Delft University of Technology, Else Kooi Laboratory, 2628 Delft, The Netherlands \\ ${ }^{8}$ Dipartimento di Fisica, Università di Trento, 38123 Povo (TN), Italy
}

(Received 22 June 2016; revised manuscript received 26 August 2016; published 8 September 2016)

\begin{abstract}
In the context of a recoil damping analysis, we have designed and produced a membrane resonator equipped with a specific on-chip structure working as a "loss shield" for a circular membrane. In this device the vibrations of the membrane, with a quality factor of $10^{7}$, reach the limit set by the intrinsic dissipation in silicon nitride, for all the modes and regardless of the modal shape, also at low frequency. Guided by our theoretical model of the loss shield, we describe the design rationale of the device, which can be used as effective replacement of commercial membrane resonators in advanced optomechanical setups, also at cryogenic temperatures.
\end{abstract}

DOI: 10.1103/PhysRevB.94.121403

Since the first demonstrations of use in an optical cavity $[1,2]$, membrane resonators have widely spread in optomechanical experiments, both as isolated mechanical oscillators and as components of hybrid systems. Their striking optical and mechanical properties allowed the observation of quantum effects induced by optomechanical interaction in the behavior of nano-oscillators [3] and in the properties of radiation itself [4].

Currently, membrane-based resonators represent a flexible tool for a wide range of scientific and technological goals: interfacing radiation at very different wavelengths $[5,6]$, implementing hybrid mechanical-atomic systems [7], fixing significant constraints on quantum gravity theories [8], and studying multimode optomechanical systems in the quantum regime [9]. These developments motivate a strong commitment to improving the performance of membrane-based oscillators. We address here the issue of mechanical losses in high stress silicon nitride ( $\mathrm{SiN}$ ) membranes, proposing a perspective which allows us to realize a "loss shield" for the membrane resonator.

SiN membrane-based devices have many mechanical resonances with frequencies starting from $0.1 \mathrm{MHz}$, with intrinsic losses well described by a model [10] where the elastic constant $K$ includes an imaginary part, $K=k(1+i \phi)$, with $\phi=1 / Q$ the loss angle and $Q$ the quality factor. Though the intrinsic quality factor is in the range $10^{6}-10^{8}$, depending on dimensions and temperature, the loss through the supporting substrate can reduce this figure by several orders of magnitude. This phenomenon is more pronounced for the lower frequency resonances, which would be the most suitable for the experimental optomechanics as higher order resonances are surrounded by numerous neighboring resonances [11]. An additional problem is the poor reproducibility of the loss

\footnotetext{
*bonaldi@science.unitn.it
}

contributed by the support, which depends on the mounting details [12], and by the loss in the sample holder [13], and it is known that these losses can sometimes be reduced by minimizing the contact of the chip frame with the sample mount [2].

The loss through the supporting substrate is usually evaluated from the energy transfer rate mediated by phonons tunneling from the membrane resonator into the substrate [14,15], an approach that motivated the development of isolation systems based on phononic band-gap shields [9,16,17]. However, this theory cannot provide precise guidelines for the design of more effective supporting systems, as it is based on some strong assumptions. In fact, the substrate is described as an infinite half-plane, so its real modal structure is not considered. Moreover, the energy transfer is unidirectional, from the membrane to the substrate. Consequently, the loss calculated within this framework does not contain the loss angle of the mechanical resonances of the substrate, and therefore no penalty is expected by the use of lossy materials.

In this Rapid Communication we rely on a coupled oscillators model, in which the vibrations of the membrane are naturally combined with those of the support to give extended normal modes. Consequently the displacement field is distributed throughout the system and the loss at the membrane resonant frequency may be substantially degraded by the contribution of the support. This effect is called recoil damping and was first studied in suspension systems for interferometric detectors of gravitational waves [18]. In this context the effective quality factor $\bar{Q}_{m}$ of a membrane resonator supported by a wafer can be approximated as

$$
\left(\bar{Q}_{m}\right)^{-1}=Q_{m}^{-1}+Q_{w}^{-1} \frac{M_{m}}{M_{w}} \frac{\omega_{w}^{2} \omega_{m}^{2}}{\left(\omega_{w}^{2}-\omega_{m}^{2}\right)^{2}},
$$

where $Q_{m}, M_{m}, \omega_{m}\left(Q_{w}, M_{w}, \omega_{w}\right)$ are the intrinsic quality factors, mass, and resonant angular frequency of the membrane (supporting wafer). According to this equation, a strong 
reduction of the quality factor is expected when a resonance of the support wafer approaches the resonance of the membrane, and the quality factor of the wafer oscillator $Q_{w}$ contributes to setting the effective quality factor, in agreement with the experimental data reported in Refs. $[13,19]$. If we consider that the recoil force due to the membrane is just the oscillating momentum $M_{m} \ddot{x}_{\mathrm{c} . \mathrm{m}}$, where $x_{\mathrm{c} . \mathrm{m} .}(t)$ is the position of the center of mass of the membrane, we can easily understand another aspect of the behavior of membrane-based resonators. In fact, it is common to observe a high quality factor for high frequency (i.e., high order) vibrations in commercial membranes. This is due to the increasingly large number of nodal lines, which averages out the contribution of different parts of the membrane in the evaluation of the center of mass, causing a strong reduction of the oscillating momentum and therefore of the recoil losses. We note that in modal shapes with an equal number of nodes and antinodes the center of mass is at rest, therefore these modes couple with the supporting wafer only through angular momentum, and the same reasoning can be repeated with moment of inertia in place of mass and recoil torque in place of recoil force.

Given that this approach reproduces the main features of the system, we have extended this theory to treat a multioscillator case, developing a scheme in which the contribution of the support to the effective quality factor of the membrane is greatly reduced. Following this design we have then produced a membrane resonator equipped with a specific on-chip structure working as a loss shield for the membrane. In this device also the low frequency vibrations of the membrane have a high quality factor and reach the limit set by the intrinsic dissipation. Figure 1(a) shows an optical microscope picture of the $\mathrm{SiN}$ membrane, with diameter $1.64 \mathrm{~mm}$, thickness $100 \mathrm{~nm}$, and internal stress $\sim 0.8 \mathrm{GPa}$. This choice of thickness allows for a nearly optimal optical coupling at a wavelength of $1064 \mathrm{~nm}$ [20]. The membrane is supported by a silicon cylinder of diameter $2.4 \mathrm{~mm}$ and thickness $1 \mathrm{~mm}$. This frame is supported in four points by a structure made of alternating flexural and torsional springs with thickness $250 \mu \mathrm{m}$. This allows an oscillatory motion of the cylinder with minimal deformation during the displacement, in order to reduce the coupling of the internal resonances of the cylinder with the rest of the structure [21,22]. As shown in Fig. 1(d), this cylinder-springs module is repeated two more times to obtain the desired degree of mechanical rigidity. In Figs. 1(e) and 1(f) we show the planar displacement of the lowest frequency resonances of this structure, respectively, at 32 and $47 \mathrm{kHz}$. It is stiffer than standard silicon frames [23] and ensures a good thermal stability when some power is dissipated in the membrane [24]. The outer frame of the device, with thickness $1 \mathrm{~mm}$, has a square shape $14 \times 14 \mathrm{~mm}^{2}$ and is clamped in a metallic holder with a central hole, which leaves uncovered the circular area of diameter $10 \mathrm{~mm}$ shown in Fig. 1(d). The device is realized by through-thickness fabrication of a silicon-oninsulator wafer with standard microelectromechanical systems technology [22,25].

In a constrained circular membrane with a high intrinsic tensile stress $\sigma_{m}$, the theoretical resonance frequencies are given by the expression $f_{n k}=f_{0} \alpha_{n k}$ where $\alpha_{n k}$ is the $k$ th root of the Bessel polynomial of order $n$, and $f_{0}=\frac{1}{2 \pi R} \sqrt{\frac{\sigma_{m}}{\rho_{m}}}$, with
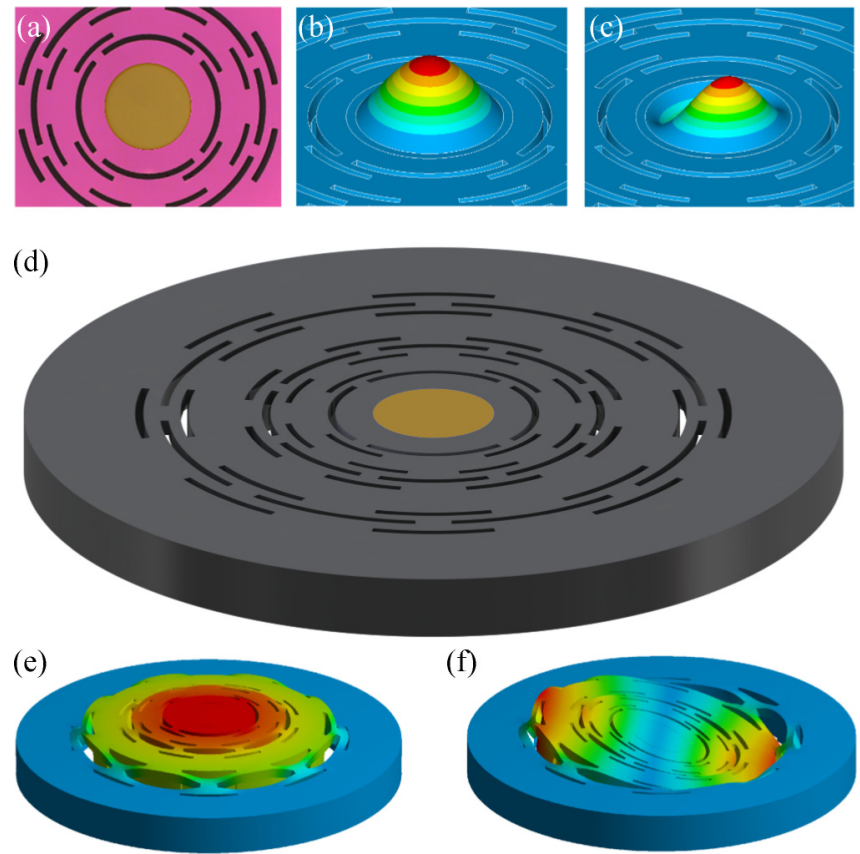

FIG. 1. (a) Optical microscope picture of the circular membrane, with diameter $1.6 \mathrm{~mm}$ and thickness $100 \mathrm{~nm}$. (b) and (c) First modal shapes involving the membrane, resonating at about 230 and $366 \mathrm{kHz}$, respectively, similar to the constrained membrane's normal mode $(n, k)=(0,1)$ and $(n, k)=(1,1)$. (d) CAD image of the device. (e) and (f) Modal shapes of the lowest frequency resonances of the device, respectively, at 32 and $47 \mathrm{kHz}$, where the planar displacement of the membrane frame can be seen.

$\rho_{m}$ the density and $R$ the radius of the membrane. The index $n$ may assume the values $(0,1, \ldots)$ and sets the number of nodal diameters of the normal mode; the index $k$ may assume the values $(1,2, \ldots)$ and sets the number of nodal circumferences. The finite element shapes shown in Figs. 1(b) and 1(c) are nearly equivalent to the normal modes $(n, k)=(0,1)$ and $(n, k)=(1,1)$ of the constrained membrane. We note that the effective modal mass of the $(0, k)$ modes for a centered, $\delta$-like readout decreases at higher values of $k$, because the absolute displacement of the modal shapes becomes more concentrated in the center [25]. In an optomechanical setup it is therefore possible to improve the coupling with the light by focusing the laser beam at the center of the membrane, implementing a nearly optimal readout. For comparison, in a square membrane the effective mass remains constant as the modal indexes change.

To evaluate the effective quality factor of the membrane in the strong-coupling model, we first identify three mechanical oscillators in the system: the membrane resonator, the supporting wafer, and an intermediate oscillator acting as loss shield, as shown in Fig. 2(a). Each oscillator is described by its frequency $v_{i}$ and mass $M_{i}$, where the subscript $i$ can be $(m, s, w)$ to identify, respectively, membrane, shield, or wafer oscillators. We describe the loss in each part by an imaginary component of the spring constant, $K_{i}=k_{i}\left(1+i / Q_{i}\right)$, where $k_{i}=4 \pi^{2} v_{i}^{2} M_{i}$ and $Q_{i}$ is the quality factor assigned to each oscillator. For a high-stress membrane of this size the intrinsic quality factor is $Q_{m} \simeq 10^{7}$, while the loss properties of the 
(a)

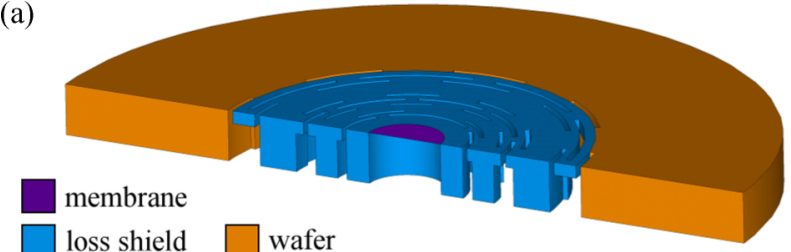

(b)

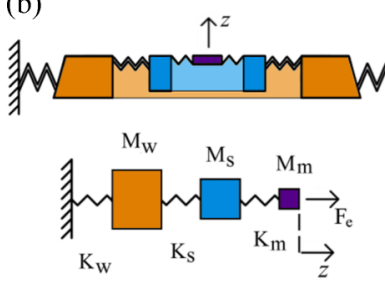

(d)
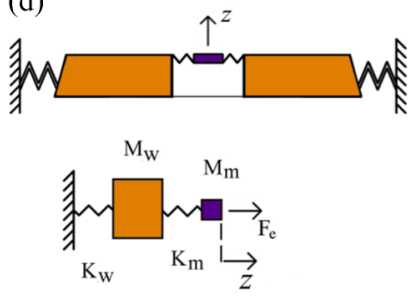

(c)

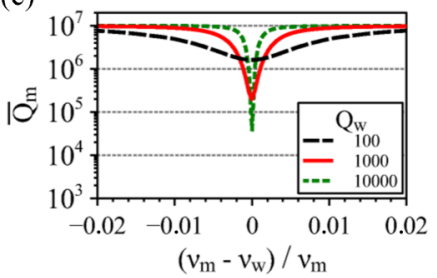

(e)

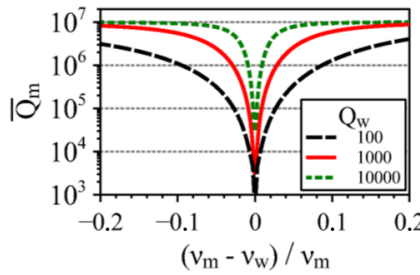

FIG. 2. (a) Section of the device where different colors denote the membrane resonator, the support wafer, and the intermediate oscillator acting as loss shield. (b) Schematic of the mechanical system and pointlike mass model. (c) Plot of the main membrane mode effective quality factor $\bar{Q}_{m}$ when a wafer mode is located at a frequency $v_{w}$ close to the membrane resonance $v_{m}$. (d) Schematic of the mechanical system and pointlike mass model of a device without loss shield structure. (e) Plot of the main membrane mode effective quality factor $\bar{Q}_{m}$ without loss shield, showing an "influence band" about 30 times larger.

clamped area are determined by the losses induced by the sample holder, and it is common to observe $Q_{w}$ in the range $10^{2}-10^{3}$. The quality factor $Q_{s}$ of the intermediate structures can vary from $10^{3}$ to some $10^{4}$, depending on the vibrational shape. In Fig. 2(b) we see the schematic of the device and the pointlike mass model used for the evaluation of the mechanical loss. The corresponding vibrational shapes of membrane and shield can be seen in Figs. 1(b) and 1(e), respectively. Given that in the coupled system it is no longer possible to distinguish the individual oscillators, the membrane resonator is identified exactly as in an experiment, that is, looking for the vibration of the system with frequency and effective mass closest to that expected. We stress that this method estimates exactly the quality factor measured in a dynamic experiment (response to an excitation or free decay) or in the thermal noise spectrum. We define this observed system as "coupled membrane," while the membrane uncoupled oscillator corresponds to the normal mode of the constrained circular membrane. Therefore we calculate the dynamic response of the system to a harmonic forcing $F_{e}$ applied to the membrane, and assess the quality factor $\bar{Q}_{m}$ from the linewidth [26].

In the graph of Fig. 2(c) we used the typical parameters of the $(0,1)$ membrane oscillator $\left(v_{m}=250 \mathrm{kHz}\right.$, $\left.M_{m}=1.5 \times 10^{-10} \mathrm{~kg}, Q_{m}=10^{7}\right)$ and of the shield oscillator $\left(v_{s}=30 \mathrm{kHz}, M_{s}=1 \times 10^{-5} \mathrm{~kg}, Q_{s}=10^{3}\right)$. Instead we vary the frequency of the wafer oscillator, with mass
$M_{w}=5 \times 10^{-5} \mathrm{~kg}$, in the neighborhood of $v_{m}$, where $\Delta v / v=$ $\left(v_{m}-v_{w}\right) / v_{m}$ is the relative frequency shift. We assign three different values to the loss angle of the wafer $1 / Q_{w}$. It is evident that, until the wafer oscillator frequency is far enough from the membrane frequency, the quality factor $\bar{Q}_{m}$ remains very close to the value assigned $Q_{m}$. The loss angle of the wafer oscillator becomes important when the two frequencies get closer. In fact, we see that with $Q_{w}=10^{3}$ we have $\bar{Q}_{m} / Q_{m}<$ 0.9 (corresponding to a $10 \%$ decrease of the quality factor) when $\Delta v / v<0.01$, but with $Q_{w}=10^{2}$ we have the same decrease when $\Delta v / v<0.035$. The effectiveness of this design is evident from the comparison with the standard situation, without loss shield and the membrane directly supported by the wafer [Fig. 2(d)]. As shown in Fig. 2(e), with $Q_{w}=10^{3}$, we have $\bar{Q}_{m} / Q_{m}<0.9$ within a quite large influence band $\Delta v / v<0.3$, that enlarges to $\Delta v / v<0.6$ if $Q_{w}=10^{2}$. This figure makes clear that a wafer oscillator with very low $Q_{w}$ can increase the loss of the membrane oscillator even if it is relatively distant in frequency. Since the quality factor of the wafer's vibrations is determined also by the sample holder, this recoil losses model explains the great influence of the assembly and the reproducibility issues. Moreover, the use of the loss shield can improve $\bar{Q}_{m}$ by many orders of magnitude (with $\bar{Q}_{m}<Q_{m}$ ) when the wafer and membrane vibrations are nearly at resonance, as can be seen by comparing Figs. 2(c) and 2(e) [26].

This reasoning can be extended to all of the modal shapes of the coupled membrane oscillator, as the device naturally profits by the various modes of the intermediate elastic structure: for instance, the resonance shown in Fig. 1(c) couples with the support through recoil torque and is shielded by the mode of Fig. 1(f). This ensures an efficient shielding for all of the resonances of the coupled membrane, starting with the lowest frequency up to a few MHz. In fact, even if the occurrence of wafer and structure modes with frequency coinciding to the resonances of the coupled membrane should be avoided (the shield does not completely eliminate the coupling loss in this case), the probability of spoiling a substantial number of coupled membrane resonances is much reduced by the use of the filter, thanks to the reduction by a factor of 30 of the influence band. Moreover, the vibrations of the membrane are coupled mainly with the inner cylinder, which features a moderate modal density of about ten modes/MHz up to a few $\mathrm{MHz}$.

The coupled membrane resonances can be seen in the thermal power spectrum density of the membrane displacement [Fig. 3(a)], acquired using a Michelson interferometer with the sample kept in a vacuum chamber. The frequencies of all resonances are in good agreement with the modal frequencies of the constrained membrane, if we set $\sigma_{m} / \rho_{m}=$ $2.4 \times 10^{5} \mathrm{~N} \mathrm{~m} / \mathrm{kg}$. To measure the quality factor we drive the system by a piezoelectric actuator mounted on the sample holder and measure the free decay time of each coupled membrane resonance. In Fig. 3(b) we plot, for the main device shown in Fig. 1, the quality factor of all resonances up to a frequency of $2 \mathrm{MHz}$. All vibrations with a frequency higher than $0.5 \mathrm{MHz}$ fulfill the minimum requirement for quantum optomechanics at room temperature, $\bar{Q}_{m}>6 \times 10^{12} / v_{m}$ [27]. We show also the quality factor of a test device with a membrane of the same size but featuring a shield oscillator resonating at about $90 \mathrm{kHz}$ (about three times the value chosen 


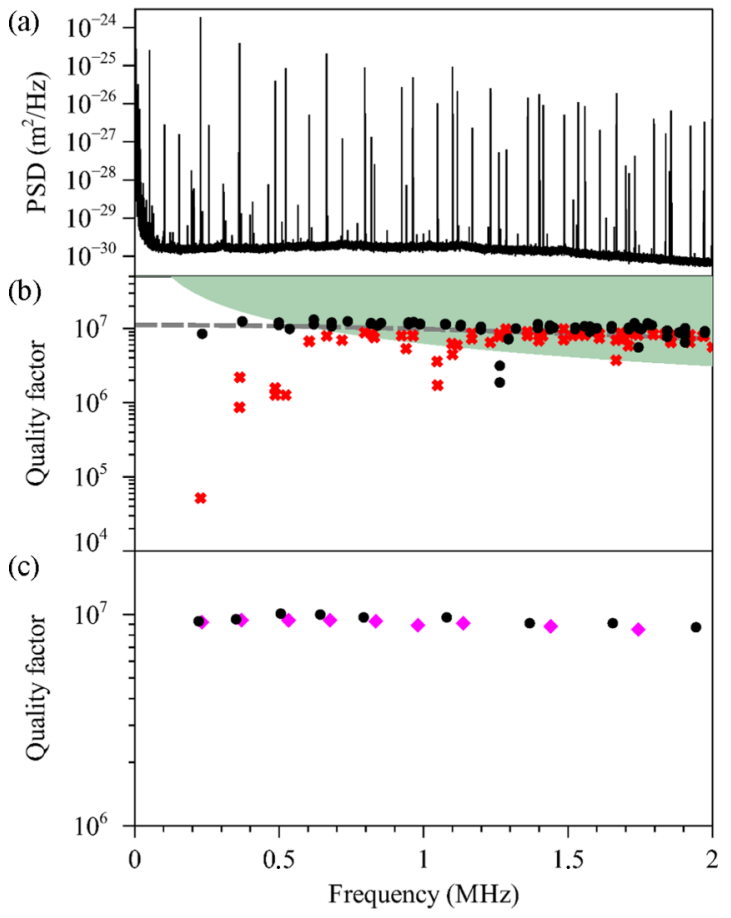

FIG. 3. (a) Power spectrum density of the displacement noise of the membrane, measured with a resolution bandwidth of $30 \mathrm{~Hz}$. Some electrical and seismic lines can be seen especially below $1 \mathrm{MHz}$. The amplitude of coupled membrane resonances is compatible with thermal force noise driving. (b) Measured quality factor of each resonance. Closed circles: main device; crosses: test device with reduced loss shield. The dashed line shows the quality factor envelope due to intrinsic loss for a square membrane with side $1.52 \mathrm{~mm}$ and fundamental frequency $230 \mathrm{kHz}$, evaluated [28] with a loss angle $\phi=1.25 \times 10^{-4}$. The data points within the shaded area fulfill the minimum requirement for quantum optomechanics at room temperature, $\bar{Q}_{m}>6 \times 10^{12} / v_{m}$ [27]. (c) Quality factor at $4.3 \mathrm{~K}$ for two samples, with slightly different intrinsic stress. At this temperature the requirement for quantum optomechanics is surpassed by more than one order of magnitude.

for the main device shown in Fig. 1). As explained in the Supplemental Material [26], this shield is much less effective in preserving the intrinsic quality factor of the membrane. In both cases the results confirm our recoil losses analysis. For the main device, the quality factor measured at low frequency is compatible with the value calculated for a square membrane with the same fundamental frequency $[10,28]$. In Fig. 3(c) we show the quality factor of some coupled membrane resonances at $4.3 \mathrm{~K}$, corresponding to $(0, k)$ and some $(1, k)$ modes of the constrained membrane. The frequency dependence of the quality factor remains smooth at low temperature, confirming that the membrane is not influenced by changes in the mechanical properties of the sample holder [29]. These results confirm the predictive value of our model and its practical effectiveness. In comparison with unshielded membranes [28,30], our device reaches the limit set by the intrinsic dissipation starting from the low frequency resonances and regardless of the modal shape, independently from the experimental setup and with a clamping system suitable for the cryogenic use.

Within the recoil losses framework we can also estimate the quality factor of membranes with phononic shields, where the wafer becomes a periodic structure with band gaps $[16,17]$. In this case membrane vibrations with frequency within the band gap have demonstrated high quality factors independently of the clamping system. In our context, with a band gap of about $0.3 \mathrm{MHz}$, the frequency shift between a membrane resonance at $3 \mathrm{MHz}$ and structure modes is $\Delta v / v \simeq 0.05$. As a rough estimate, from Fig. 2(e) we can expect a reduction of about $50 \%$ of the quality factor in the case $Q_{w}=1000$, which is a good result but still not optimal. However, better results can be obtained by widening the band gap or by cooling the sample at cryogenic temperatures, where the influence bands shrink thanks to a general improvement in the wafer's quality factor. Unfortunately the extension of this technique to the $100 \mathrm{kHz}$ range would require the use of very large isolation structures. In fact, a band gap centered at a frequency $f=100 \mathrm{kHz}$ can be obtained with a $10 \times 10$ array of unit cells with characteristic length $v /(2 f) \simeq 10 \mathrm{~mm}$, where $v$ is the sound velocity in silicon.

All things considered, our recoil losses analysis allows one to build robust devices with high quality factor, preserving the thermal and geometrical characteristics of typical membrane-based resonators. The optical properties of these membranes are compatible with their use as optomechanical oscillators [27], both in Michelson interferometers and in cavity setups [25]. Since their quality factor is high in the whole frequency range, they can be used with optimal efficiency, both in single-mode applications, such as optical cooling [31], and in multimode applications, such as hybridization [32] and two-mode squeezing [33]. For these reasons we imagine that this class of devices may spread as an effective replacement of standard membrane resonators in advanced optomechanical setups, also at cryogenic temperatures.

This work has been supported by MIUR ("PRIN 20102011" and "QUANTOM") and by INFN ("HUMOR" project). A.B. acknowledges support from the MIUR under the "FIRBFuturo in ricerca 2013" funding program, project code RBFR13QUVI.
[1] J. D. Thompson, B. M. Zwickl, A. M. Jayich, F. Marquardt, S. M. Girvin, and J. G. E. Harris, Nature (London) 452, 72 (2008).

[2] D. J. Wilson, C. A. Regal, S. B. Papp, and H. J. Kimble, Phys. Rev. Lett. 103, 207204 (2009).

[3] T. P. Purdy, R. W. Peterson, and C. A. Regal, Science 339, 801 (2013).
[4] T. P. Purdy, P.-L. Yu, R. W. Peterson, N. S. Kampel, and C. A. Regal, Phys. Rev. X 3, 031012 (2013).

[5] R. Andrews, R. Peterson, T. Purdy, K. Cicak, R. Simmonds, C. Regal, and K. Lehnert, Nat. Phys. 10, 321 (2014).

[6] T. Bagci, A. Simonsen, S. Schmid, L. G. Villanueva, E. Zeuthen, J. Appel, J. M. Taylor, A. Sørensen, K. Usami, A. Schliesser, and E. S. Polzik, Nature (London) 507, 81 (2014). 
[7] A. Jöckel, A. Faber, T. Kampschulte, M. Korppi, M. T. Rakher, and P. Treutlein, Nat. Nanotechnol. 10, 55 (2015).

[8] M. Bawaj, C. Biancofiore, M. Bonaldi, F. Bonfigli, A. Borrielli, G. Di Giuseppe, L. Marconi, F. Marino, R. Natali, A. Pontin, G. A. Prodi, E. Serra, D. Vitali, and F. Marin, Nat. Commun. 6, 7503 (2015).

[9] W. H. P. Nielsen, Y. Tsaturyan, C. B. Møller, E. S. Polzik, and A. Schliesser, arXiv:1605.06541.

[10] L. G. Villanueva and S. Schmid, Phys. Rev. Lett. 113, 227201 (2014).

[11] Nearby modes can interfere with the measurement by increasing the displacement background noise or by coupling with the main mode through the intracavity field.

[12] T. P. Purdy, R. W. Peterson, P.-L. Yu, and C. A. Regal, New J. Phys. 14, 115021 (2012).

[13] S. Schmid, K. D. Jensen, K. H. Nielsen, and A. Boisen, Phys. Rev. B 84, 165307 (2011).

[14] I. Wilson-Rae, Phys. Rev. B 77, 245418 (2008).

[15] I. Wilson-Rae, R. A. Barton, S. S. Verbridge, D. R. Southworth, B. Ilic, H. G. Craighead, and J. M. Parpia, Phys. Rev. Lett. 106, 047205 (2011).

[16] P.-L. Yu, K. Cicak, N. S. Kampel, Y. Tsaturyan, T. P. Purdy, R. W. Simmonds, and C. A. Regal, Appl. Phys. Lett. 104, 023510 (2014).

[17] Y. Tsaturyan, A. Barg, A. Simonsen, L. G. Villanueva, S. Schmid, A. Schliesser, and E. S. Polzik, Opt. Express 22, 6810 (2014).

[18] P. R. Saulson, Phys. Rev. D 42, 2437 (1990).

[19] A. Jöckel, M. T. Rakher, M. Korppi, S. Camerer, D. Hunger, M. Mader, and P. Treutlein, Appl. Phys. Lett. 99, 143109 (2011).

[20] M. Born and E. Wolf, Principles of Optics (Pergamon, New York, 1989).
[21] E. Serra, A. Borrielli, F. S. Cataliotti, F. Marin, F. Marino, A. Pontin, G. A. Prodi, and M. Bonaldi, Appl. Phys. Lett. 101, 071101 (2012).

[22] A. Borrielli, A. Pontin, F. S. Cataliotti, L. Marconi, F. Marin, F. Marino, G. Pandraud, G. A. Prodi, E. Serra, and M. Bonaldi, Phys. Rev. Appl. 3, 054009 (2015).

[23] A standard $5 \times 5 \mathrm{~mm}^{2}$ silicon frame with thickness $500 \mu \mathrm{m}$ resonates at $\simeq 20 \mathrm{kHz}$ when supported by a side or at $\simeq 16 \mathrm{kHz}$ when fixed at one corner.

[24] The expected temperature increase of the central cylinder is estimated as $4.5 \mathrm{mK} / \mathrm{mW}(21 \mathrm{mK} / \mathrm{mW})$ at $300 \mathrm{~K}(4.2 \mathrm{~K})$, far smaller than the unavoidable internal gradient setting up in the nanometric (and less conductive) SiN membrane.

[25] E. Serra et al., AIP Advances 6, 065004 (2016).

[26] See Supplemental Material at http://link.aps.org/supplemental/ 10.1103/PhysRevB.94.121403 for more details.

[27] M. Aspelmeyer, T. J. Kippenberg, and F. Marquardt, Rev. Mod. Phys. 86, 1391 (2014).

[28] P.-L. Yu, T. P. Purdy, and C. A. Regal, Phys. Rev. Lett. 108, 083603 (2012).

[29] The measurements also suggest that the loss angle of $\mathrm{SiN}$ remains unchanged, even though the interplay with possible additional effects, related to $\mathrm{SiN}$ growth parameters or to microfabrication details, should be carefully investigated.

[30] S. Chakram, Y. S. Patil, L. Chang, and M. Vengalattore, Phys. Rev. Lett. 112, 127201 (2014).

[31] R. W. Peterson, T. P. Purdy, N. S. Kampel, R. W. Andrews, P.-L. Yu, K. W. Lehnert, and C. A. Regal, Phys. Rev. Lett. 116, 063601 (2016).

[32] A. B. Shkarin, N. E. Flowers-Jacobs, S. W. Hoch, A. D. Kashkanova, C. Deutsch, J. Reichel, and J. G. E. Harris, Phys. Rev. Lett. 112, 013602 (2014)

[33] Y. S. Patil, S. Chakram, L. Chang, and M. Vengalattore, Phys. Rev. Lett. 115, 017202 (2015). 